\title{
Enabling PTAs for gravitational wave detection: The all-sky HTRU pulsar survey
}

\section{Cherry $\mathbf{N g}^{1}$}

Max-Planck-Institut für Radioastronomie

Auf dem Hügel 69, 53121 Bonn, Germany

E-mail: cherryng@mpifr-bonn.mpg . de

\section{Ewan Barr ${ }^{1}$}

Max-Planck-Institut für Radioastronomie

Auf dem Hügel 69, 53121 Bonn, Germany

E-mail: ebarrampifr-bonn.mpg.de

\begin{abstract}
The extreme conditions found in and around pulsars make them fantastic natural laboratories for probing many aspects of fundamental physics and astronomy. One of the most interesting applications of pulsar astronomy is the detection of gravitational waves through highly accurately-timed millisecond pulsars (MSPs) in a timing array. To discover more MSPs we have begun the High Time Resolution Universe (HTRU) survey; a blind survey of the northern sky with the 100-m Effelsberg radio telescope complemented by a twin survey of the southern sky with the 64-m Parkes telescope. The surveys use state-of-the-art multi-beam receivers and high time and frequency resolution backends, to probe deeper into the Galaxy than ever before. The surveys will cover the whole sky including the region north of $38^{\circ}$ Galactic latitude which was previously almost unsearched by the pulsar community. Based on simulations, we expect to discover about 100 MSPs in this region. The southern survey started in early 2008, and the first northern survey pointings were taken in the summer of 2010. Thus far, the surveys have already detected five new high-dispersion measure MSPs that may be of use as part of a timing array. The combined efforts of both northern and southern surveys will lead to complete sky coverage with a sensitivity and speed previously unachievable, shedding light on exciting science and aiding gravitational wave detection efforts.
\end{abstract}

25th Texas Symposium on Relativistic Astrophysics -TEXAS2010

Heidelberg, Germany

December $06-112010$

1 On behalf of the HTRU consortium, http://astronomy. swin.edu.au/pulsar/?topic=hlsurvey. 


\section{Introduction}

Pulsars are highly magnetised, rapidly rotating neutron stars that radiate a beam of coherent radio emission from the magnetic poles. The extreme conditions found in and around pulsars make them fantastic natural laboratories, providing insights to a rich variety of aspects of fundamental physics and astronomy. One of the most interesting applications of pulsar astronomy is the detection of gravitational waves through highly precisely-timed millisecond pulsars (MSPs: pulsars with rotation periods below $20 \mathrm{~ms}$ ) in a timing array (e.g. [1]). The very rapidly rotating MSP is the pulsar of choice when it comes to high precision measurements, as the exceptional rotational stability of MSPs is generally 3 to 4 orders of magnitude better than normal pulsars. [2] has shown that the stochastic gravitational wave background, as predicted from hierarchical galaxy formation models, could be detected directly with 5 years of observations of 20 to 40 MSPs timed with a precision of 100 nanoseconds.

To discover more MSPs, we have begun the High Time Resolution Universe (HTRU) survey; a blind survey of the northern sky with the 100-m Effelsberg radio telescope and a twin survey of the southern sky with the 64-m Parkes telescope [3]. The surveys use multi-beam receivers and backends constructed with recent advancements in technology, providing unprecedentedly high time and frequency resolution. The combined efforts of both northern and southern surveys will lead to complete sky coverage with a sensitivity and speed previously unachievable, enabling a host of scientific experiments and aiding gravitational wave detection efforts. In this publication, we summarise the scientific motivations, the current status of the HTRU survey and its recent successes.

\section{Motivations of the HTRU survey}

A blind all-sky search is the only way to significantly increase the number of known pulsars in an unbiased way and to gain a better understanding of the population of MSPs. The HTRU survey will cover the whole sky and including the region north of $38^{\circ}$ Galactic latitude which has never been searched at high frequencies for MSPs previously. HTRU will be the most comprehensive all-sky survey for pulsars and fast transient sources to date, creating a petabyte of digital data. Furthermore, the deteriorating RFI environment makes this the best time to conduct such a survey, until the next generation of radio telescopes, such as the Square Kilometre Array (SKA), come on-line.

While the previous Parkes multi-beam surveys for pulsars (e.g. [4]) were cutting-edge for that time, the early Parkes surveys employed analogue filterbanks with 3-MHz channel width, providing relatively low frequency resolution. This becomes a limitation when we consider the dispersive nature of observed pulsar radiation. As the pulses travel to the Earth through the ionized interstellar medium, lower frequency radio waves arrive later than the higher frequency counterparts over a finite frequency bandwidth. Such dispersion delay within a single filterbank channel can smear out the pulses and reduce our sensitivity, particularly affecting high distant pulsars. This can partially explain why MSPs have been mostly discovered only in the Galactic neighbourhood. In addition, the data of the previous Parkes surveys were taken with 1-bit 
digitization and a modest time resolution of $250 \mu$ s. These have imposed limitations on probing the true boundaries of pulsar parameter space.

In the current HTRU survey, the construction of tunable digital filterbanks has made it possible to create fine filters with a frequency resolution of $0.5 \mathrm{MHz}$. The higher frequency resolution will help the negation of interstellar dispersion, and will enable the survey to be capable of detecting distant, highly-dispersed MSPs within the Galaxy. This improvement in frequency resolution is particularly exciting for Galactic plane observations, finding previously hidden pulsars in this part of the Galaxy where dispersion measures are the highest. In addition, faster sampling has also been implemented in the HTRU survey. The HTRU-North is sampled every $53 \mu$ s and the HTRU-South every $64 \mu \mathrm{s}$. This will enable characterisation of the transient sky on short timescales.

\section{Observing strategy}

The pulsar population is not evenly distributed in the sky. While younger pulsars are more likely to be found close to their birth place in the Galactic plane, the older MSPs have more time to move to higher Galactic latitudes. In addition, due to the influence of propagation effects of the interstellar medium [5], there is a strong dependence of pulsar signal with respect to the Galactic latitude. To obtain maximum yield and efficiency for different regions of the Galaxy, pointing integrations have been optimised accordingly based on simulations and experiences from past surveys. The HTRU survey is conducted in three distinct observing regimes, and table 1 provides a summary of the specifications.

\subsection{Deep Galactic plane survey for relativistic binaries}

The Galactic plane with its wealth of pulsars offers the best chance at finding the most extreme and exciting systems that the Milky Way has to offer. The Galactic plane is expected to host as many as 100 double neutron star systems [6], some of which are expected to be even more compact than the double pulsar system [7]. Such systems do not receive a large kick velocity from either of the supernovae explosion wherein they were formed, and hence are most likely to be found close to the Galactic plane where they are born. The survey has the potential to discover pulsar-black hole systems, which is of great interest as their strong gravitational field would provide the best tests for general relativity. In order to probe a larger volume of the Galaxy and to achieve the required sensitivity for the detection of more distant systems, the longest integration is employed in the deep Galactic plane survey within the region of $|b|<3.5^{\circ}$. Pointings comprise 25 minute integrations in the HTRU-North and 72 minutes for the HTRUSouth, which is double that of the previous Galactic plane surveys.

\subsection{Mid-latitudes survey for MSPs as timing array sources}

At $|b|<15^{\circ}$ Galactic latitudes, prior surveys discovered a number of bright, but previously undetected MSPs. These types of discovery are essential to the success of projects such as pulsar timing arrays, where a large number of MSPs separated by a variety of angular distances is required. Pointings in the mid-latitude survey will comprise 3 minute integrations for HTRU- 
North in the region $3.5^{\circ}<|b|<15^{\circ}$. For HTRU-South the integrations are again double that of the previous survey to 9 minutes per pointing.

\subsection{All-sky survey for sub-millisecond pulsars and transients}

In the 20 years since the last northern-sky survey, not only have we enhanced our search capabilities, but we have also become aware of the existence of transient signals [8], some of which may be of extragalactic origin [9]. Here we adapt our survey to search the full sky for transient sources. Furthermore, this survey will place the best constraints on the existence of sub-millisecond pulsars. If such sources do exist, the HTRU survey at high-latitude will have the best chances yet for the detection, benefited by the extremely low dispersion measure in this region. Such a discovery would certainly provide new constraints on the equations of state for dense nuclear matter. Pointings in the high-latitude survey will comprise 1.5 minute integrations for HTRU-North and 4.5 minute integrations for HTRU-South in the region $|b|>15^{\circ}$.

Table 1. Summary of the specifications of the HTRU-North and South survey:

\begin{tabular}{|l|l|l|}
\hline & Northern survey & Southern survey \\
\hline Start date: & Summer 2010 & Early 2008 \\
\hline Telescope: & Effelsberg-100m & Parkes-64m \\
\hline Sky coverage: & $\delta>0$ & $\delta<+10^{\circ}$ \\
\hline & Low-lat: $1500 \mathrm{~s}$ & Low-lat: 4300 s \\
Integration time: & Mid-lat: $180 \mathrm{~s}$ & Mid-lat: $540 \mathrm{~s}$ \\
& High-lat: $90 \mathrm{~s}$ & 13-beam 1.35-GHz receiver \\
\hline Receiver: & 7-beam 1.4-GHz receiver & Berkeley-Parkes-Swinburne \\
\hline Backend: & Pulsar Fast Fourier Transform & Recorder (BPSR) \\
\hline Bandwidth: & Spectrometer (PFFTS) & $340 \mathrm{MHz}$ \\
\hline No. of channels: & $300 \mathrm{MHz}$ & 1024 \\
\hline Freq resolution: & 512 & $0.39 \mathrm{MHz}$ \\
\hline Time resolution: & $0.58 \mathrm{MHz}$ & $64 \mu \mathrm{s}$ \\
\hline No. sky pointings: & $54 \mu \mathrm{s}$ & $\sim 43,000$ \\
\hline Data sizes: & $\sim 180,000$ & $\sim 1$ petabyte \\
\hline
\end{tabular}

\section{Current survey status}

For the southern survey, more than $50 \%$ of the high-latitude region has been observed and the mid-latitude observations have been completed. The low-latitude survey began in the beginning of 2011 and so far about $15 \%$ of the Galactic plane has been observed. Currently only about $10 \%$ of the data obtained has been processed, but already there have been 73 pulsar discoveries, of which the first 27 discoveries are described in [3]. Among these 73 new pulsars there is a high fraction of MSPs discovered (14/73 compared to the current MSP population of less than $10 \%$ of all pulsars). Furthermore, five of these new MSP discoveries are at high DM, all of which lie within previously surveyed regions of the sky. These statistics confirm the superiority of the set-up of the HTRU survey for MSP discovery. Even more MSP discoveries are expected when a search for accelerated signals (as would be observed from binary systems) 
is included in the data processing. Exotic objects like a magnetar and pulsar-planet system have also been discovered from the HTRU-South survey [10].

For the northern survey, system tests have been carried out by observing unidentified point sources from the Fermi satellite, which has led to the discovery of Effelsberg's first ever MSP in January 2010 [11]. To-date, 4\% of survey observations have been conducted and initial processing is underway.

\section{References}

[1] G. Hobbs et al., Publications of the Astronomical Society of Australia, 26, 103 (2009)

[2] F.A. Jenet et al., The Astrophysical Journal, 625, 123 (2005)

[3] M. J. Keith et al., Monthly Notices of the Royal Astronomical Society, 409, 619 (2010)

[4] R. N. Manchester et al., Monthly Notices of the Royal Astronomical Society, 328, 17 (2001)

[5] D. R. Lorimer, M. Kramer, Handbook of pulsar astronomy, Cambridge University Press, 2005

[6] K. Belczynski et al., The Astrophysical Journal, 572, 407 (2002)

[7] M. Kramer, I. H. Stairs, Annual Review of Astronomy \& Astrophysics, 46, 541 (2008)

[8] M. A. McLaughlin et al., Nature, 439, 817 (2006)

[9] D. R. Lorimer et al., Science, 318, 777 (2007)

[10] L. Levin et al., The Astrophysical Journal Letters, 721, 33 (2010)

[11] E. Barr et al., in prep. 